REOP. Vol. 13, No 1, $1^{\text {er }}$ Semestre, 2002, pp. 69-87

\title{
UN ANÁLISIS DE LAS METAS EN SITUACIÓN DE APRENDIZAJE PARA EL ALUMNADO DE PRIMARIA Y SECUNDARIA OBLIGATORIA
}

\section{ANALYSIS OF THE GOALS FOR SCHOOL CHILDREN IN PRIMARY AND SECONDARY SCHOOL}

\author{
Carlota González Gómez*, Germán Torregrosa** y Leandro Navas*** \\ Universidad de Alicante
}

\begin{abstract}
RESUMEN
Este trabajo se enmarca en dos contextos sobre la motivación por el aprendizaje escolar: la teoría atribucional de Weiner $(1986,1995)$ y las aportaciones de Dweck (1986) sobre las metas que persigue el alumnado. El objetivo general es averiguar las metas que muestran los alumnos de primaria y secundaria obligatoria en situación de aprendizaje, la relación que mantienen estas metas con las diferentes dimensiones causales y si hay diferencias entre las metas de los estudiantes de secundaria obligatoria y las de los alumnos de primaria. La muestra la componen 722 alumnos de primaria y secundaria. Los resultados obtenidos indican que las metas coinciden con las halladas en otros contextos pero que se producen diferencias en función de la etapa educativa.
\end{abstract}

Palabras clave: Metas de aprendizaje, dimensiones causales, motivación.

\section{ABSTRACT}

This work is based on two contexts regarding school learners' motivation: the atributional theory by Weiner $(1986,1995)$ and the contributions made by Dweck (1986) about the goals schoolchildren ex-

* Carlota González Gómez. Profesora Ayudante de Psicología Evolutiva y de la Educación en la Facultad de Educación de la Universidad de Alicante. Sus investigaciones y publicaciones se encuadran en la formación del personal docente y en la respuesta educativa a las necesidades educativas especiales (especialmente en lo que concierne a niños superdotados y con altas habilidades).

** Germán Torregrosa. Profesor Titular E. U. de Didáctica de la Matemática y Vicedecano de Ordenación Académica de la Facultad de Educación de la Universidad de Alicante. Sus líneas de investigación se centran en el análisis de textos, en el razonamiento en geometría, en la formación del profesorado y en la evaluación de programas educativos. Es miembro del Comité de Evaluación de la Calidad de la U.A.

*** Leandro Navas. Catedrático E. U. de Psicología Evolutiva y de la Educación y Vicedecano de Prácticas y Estudios de la Facultad de Educación de la Universidad de Alicante. Sus líneas de investigación giran en torno a los determinantes del rendimiento académico, la motivación, la evaluación educativa y la evaluación de programas educativos. Ha dirigido y participado en varios proyectos subvencionados por el CIDE. 
pect to reach. The main aim of this work is to find the goals shown by primary and secondary school students during their learning stage, the relations these goals hold with the different causal dimensions and if there exist differences between the primary schoolchildrens' goals and those of the secondary school learners. The sample we have invetigated consists of 722 students of both primary and secondary school. The results obtained indicate that their goals happen to be the same as those found in other contexts but that differences appear depending on the learning stage.

Key words: Schoolchildrens' goals, causal dimensions, motivation.

\section{Introducción}

Debido a la importancia que tiene la motivación en el aprendizaje escolar, son numerosas las teorías que han tratado de explicar, desde distintos puntos de vista, los factores que influyen sobre ella. Durante la dos últimas décadas dos aportaciones merecen ser resaltadas. De una parte, la teoría atribucional de Bernard Weiner (1986) y, por otro lado, la aproximación que se centra en las metas que persigue el alumnado (Dweck, 1986).

La teoría atribucional de la motivación de logro de Weiner identifica los aspectos cognitivos de la motivación relacionados con la atribución causal y persigue explicar y comprender cómo el sujeto juzga acontecimientos, cómo percibe la información que le va a permitir predecir o controlar su conducta y la de los demás, y cómo hace atribuciones de responsabilidad (Weiner, 1995). En contextos de logro, cuando el sujeto obtiene un resultado negativo, inesperado o muy importante inicia la búsqueda de causas para explicarlo, lo que se traduce en atribuciones a la capacidad, al esfuerzo, a la suerte o a la dificultad de la tarea. Estas atribuciones causales se relacionan con las expectativas y con las reacciones afectivas, que influyen, a su vez, en una mayor o menor motivación. Estas influencias no son realizadas directamente por las atribuciones, sino que tienen lugar, de modo indirecto, a través de las distintas dimensiones causales.

Weiner (1986) describe tres dimensiones para categorizar las atribuciones causales:

a) Dimensión de lugar, según la cual las causas pueden ser internas (si se sitúan dentro de la persona) o externas (si se ubican fuera del sujeto), de hecho: "La localización causal es una de las principales dimensiones o propiedades del pensamiento causal» (Weiner, 1995, p. 7);

b) Estabilidad, por la que surgen causas estables (que permanecen a través del tiempo y de las situaciones) o inestables (que son pasajeras), y

c) Controlabilidad, y en función de ella las causas son controlables o incontrolables (si se atribuye el resultado al esfuerzo realizado, hablaremos de una causa controlable, mientras que, si se atribuye a la suerte o a la capacidad, sería una causa incontrolable).

Son estas dimensiones, y no las causas en sí, las que determinan las expectativas, los sentimientos y la motivación de los sujetos. Todos ellos, posteriormente, repercutirán sobre el rendimiento.

En este modelo, es necesario tener en cuenta, también, los antecedentes que llevan al sujeto a realizar una determinada atribución causal. Entre los antecedentes causales se señalan: la información específica que posee el sujeto acerca de sus realizaciones, el conocimiento de las reglas de causalidad y las tendencias hedónicas. La información específica se 
deriva de la observación de los resultados de la propia conducta y de la comparación de los mismos con los obtenidos por sus compañeros. El sujeto valora su capacidad, dicha valoración afecta al proceso atribucional y al desarrollo o a la modificación de sus expectativas y de su rendimiento.

El conocimiento de las reglas causales afecta al tipo de atribución realizada. Si el sujeto asocia el éxito con la capacidad y el fracaso con la falta de ella, ante un resultado negativo, experimentará un sentimiento de frustración que originará una atribución poco adaptativa, lo que, a su vez, repercutirá de forma negativa sobre su rendimiento. Pero si el sujeto asocia el éxito a la capacidad y al esfuerzo, y el fracaso a no haberse esforzado, ante un resultado negativo, realizará una atribución adaptativa que estimulará una serie de conductas (más atención, una mayor persistencia ante las tareas, etc.) que influirán, consecuentemente, de forma positiva en su rendimiento.

Las tendencias hedónicas hacen que los sujetos desarrollen y ofrezcan a los demás una imagen positiva de sí mismos. Por esta razón, adoptan una serie de estrategias que les van a permitir internalizar los éxitos y externalizar los fracasos. De esta manera, el sujeto crea entornos mentales o reales que le permiten verificar sus autopercepciones, aunque éstas sean negativas, adoptando, en ese caso, una estrategia defensiva para mantener sus creencias. Es decir, pone en marcha mecanismos que le permiten sentirse bien y ofrecer una imagen deseable a los demás.

El proceso atribucional descrito por Weiner $(1986,1992)$ tiene consecuencias tanto a nivel cognitivo como a nivel afectivo. En el plano cognitivo, el modelo justifica las expectativas que el sujeto desarrolla en función de los resultados alcanzados y de las dimensiones causales de las atribuciones que realiza, especialmente, de la dimensión de estabilidad. La relación entre la estabilidad y las expectativas se establece en los dos sentidos: la estabilidad de las causas determina los cambios de las expectativas y, del mismo modo, la confirmación de la expectativa conduce a dimensionalizar la causa como estable. Distintos estudios han confirmado que la estabilidad causal se relaciona con los cambios en las expectativas. Se demuestra que, tras el éxito, las adscripciones a la capacidad, causa supuestamente estable, correlacionan positivamente con las expectativas, mientras que las adscripciones a la suerte, causa inestable, lo hacen negativamente; y que, ante el fracaso, las adscripciones a la capacidad tienen una correlación negativa con las expectativas de cara al futuro (Fontaine, 1974; Inagi, 1977; Heilman y Guzzo, 1978; Kojima, 1984). En el caso de la influencia de la expectativa sobre la estabilidad, se comprueba que una alta expectativa de éxito, si es seguida por un éxito, produce atribuciones a factores estables y, al contrario, una alta expectativa de fracaso, cuando es seguida por un fracaso, también produce atribuciones a factores estables. Es decir, los resultados esperados, éxitos con alta expectativa de éxito o fracasos con baja expectativa de éxito, son adscritos a causas estables, mientras que los resultados inesperados, fracasos con alta expectativas de éxito o éxitos con expectativas de fracaso, son adscritos a causas inestables (Hayashi y Yamachi, 1974).

A nivel afectivo, el modelo explica qué sentimos en función de los resultados obtenidos y de cómo pensamos, y que las atribuciones, y sus correspondientes dimensiones causales, son condiciones suficientes de las emociones. Ante un determinado resultado, se producen unos afectos dependientes del mismo, e independientes de la atribución. Si el resultado es valorado por el sujeto positivamente se siente contento, pero si el resultado es evaluado negativamente se siente triste o frustrado. Los resultados son los que provocan una reacción 
afectiva y emocional de tipo general que tiene un efecto sobre la conducta motivada. Estos estados de ánimo iniciales, unidos a los antecedentes causales, originan el tipo de atribución que el sujeto realiza y las dimensiones de la misma. Así, atribuir el éxito a una causa interna produce orgullo e incremento en la autoestima, pero atribuir el fracaso a causas internas produce vergüenza y sentimientos de culpa. Es decir, las dimensiones causales, a su vez, determinan los afectos o sentimientos (en este caso, dependientes de la atribución) y, como se ha señalado, las expectativas. Tanto unos como otros influyen en la motivación y en el rendimiento.

En nuestro contexto, no obstante, varios estudios cuestionan algunos de estos supuestos y se pone en tela de juicio el papel de las atribuciones (Navas, Castejón y Sampascual, 1996). Así Montero y Alonso (1992), cuando comparan en situaciones de aula tres modelos motivacionales (el de Weiner y el de Dweck entre otros) obtienen más apoyo para el modelo de Dweck y no logran confirmar el papel mediador de las atribuciones entre la motivación de logro y las expectativas. También se ha hallado que los alumnos más pequeños no diferencian, de forma clara, el esfuerzo y la capacidad y que las atribuciones causales de los resultados iniciales juegan un papel menor que las atribuciones referidas a las expectativas futuras. Parece que las expectativas del individuo influyen más en sus resultados futuros que las atribuciones causales de los resultados anteriores. Las expectativas son las que determinan el esfuerzo y el resultado (Castejón, Navas y Sampascual, 1993). También hay que tener en cuenta que no todos los sujetos perciben el resultado de la misma manera, ya que atienden de forma selectiva a determinados aspectos de la información que ofrece la situación (Sampascual, Navas y Castejón, 1994). Y, por último, en otros trabajos se cuestiona el papel determinante de las atribuciones y de las dimensiones causales (Navas, Castejón y Sampascual, 2000).

En relación con la otra línea de investigación que comentábamos, centrada en las metas que persigue el alumnado al aprender, destacan los trabajos realizados por Dweck (1986) que estudia el patrón de conducta de logro adaptativa de algunos sujetos y la de los que, por el contrario, muestran indefensión ante los obstáculos de aprendizaje que se les presentan. En su trabajo describe, por un lado, la conducta de los sujetos que parecen disfrutar con los retos o los desafíos, que se motivan ante ellos y persisten en el aprendizaje y, por otro lado, en contraste con los anteriores, el comportamiento que presentan los sujetos que, ante posibles desafíos o nuevos retos, muestran una conducta de evitación y de baja persistencia en la realización de la tarea propuesta, y prestan más atención a la experiencia negativa que han podido tener en situaciones similares de desafío y a la baja autoestima que les produce tener que afrontar estos obstáculos.

Inicialmente, se consideró la existencia de dos tipos de metas: metas de aprendizaje y metas de ejecución. Las primeras se caracterizan por un interés por la adquisición y el desarrollo de nuevas capacidades y conocimientos. Están más relacionadas con un patrón motivacional orientado hacia el dominio de la tarea de aprendizaje y hacia una búsqueda de todo aquello que supone un desafío, con la consiguiente activación de estrategias de aprendizaje eficaces. Las segundas, es decir, las metas de ejecución, se caracterizan por un interés por obtener juicios y valoraciones positivas sobre la propia capacidad y están relacionadas con un patrón motivacional de indefensión. El sujeto con este tipo de metas trata de evitar, a toda costa, valoraciones negativas de su capacidad, hasta tal punto que rehúye las situaciones de aprendizaje en las que no tenga, de alguna manera, asegurado el éxito, y evita todo lo que suponga un desafío o una situación nueva no controlada. Siguiendo esta línea de tra- 
bajo, diversos estudios han demostrado la existencia de estos dos tipos de metas que, a la vez, representan formas diferentes de aproximarse a la realización de las tareas de aprendizaje, conllevan distintas percepciones y creencias en relación a la propia capacidad y al esfuerzo y dejan entrever, también, distintos patrones motivacionales (Valle y Gómez, 1997; Bzuneck, 1999; Cervelló, Escartí y Balagué, 1999).

Los sujetos con metas de ejecución interpretan el fracaso como una falta de capacidad y de competencia. Esto puede producir una reacción afectiva negativa ante las tareas de aprendizaje, lo que se traduce en una falta de implicación, baja persistencia y poco interés por la búsqueda de estrategias que le permitan solventar sus dificultades (Cervelló y Santos-Rosa, 2000; González-Pienda et al., 2000). Por el contrario, los sujetos con metas de aprendizaje no se centran en las atribuciones de su fracaso, sino que intentan buscar estrategias de autorregulación que les permitan resolver sus dificultades, como, por ejemplo, las que favorecen la comprensión del nuevo material a aprender. Valoran más el aprendizaje y dedican más esfuerzo y atención a las tareas novedosas o de dificultad intermedia (Valle et al., 2000; Dweck y Leggett, 1989; Ames, 1984). Los alumnos que se orientan hacia las metas de aprendizaje ven los retos y los desafíos como algo inherente al propio proceso y, sobre todo, como una oportunidad para aprender y aumentar las propias habilidades (Hayman y Dweck, 1992; Cervelló y Santos-Rosa, 2000). Para los sujetos con metas de ejecución la satisfacción con los resultados está basada en la habilidad que ellos creen que tienen para realizar la tarea, mientras que para los alumnos con metas de aprendizaje la satisfacción está basada en el esfuerzo que tienen que realizar para conseguir el objetivo (Cervelló y Santos-Rosa, 2000; Ames et al., 1977). De todo ello se desprende que las metas académicas son determinantes muy importantes de la motivación del alumnado por las tareas escolares.

En otro orden de cosas, Hayamizu, Ito y Yohiazaki (1989) elaboraron un cuestionario para evaluar los dos tipos de metas, hasta ese momento, identificadas (metas de aprendizaje y metas de rendimiento). Sin embargo, descubren la existencia de tres tipos. Parece que dentro de las metas de rendimiento hay dos categorías:

1) Metas de refuerzo social, que suponen una tendencia a aprender con el propósito de obtener la aprobación social (de padres y de profesores) y evitar el rechazo, y

2) Metas de logro, que están relacionadas con la tendencia a aprender para obtener buenos resultados en los exámenes y avanzar en los estudios.

Posteriormente, Hayamizu y Weiner (1991) administraron la misma escala a estudiantes universitarios y hallan las mismas clases de metas (dos dentro de las de rendimiento y una de aprendizaje). Comprueban que las dos metas de rendimiento correlacionan entre sí, pero ninguna de ellas mostraba algún tipo de asociación o dependencia con las metas de aprendizaje.

En trabajos realizados en nuestro contexto, con alumnos de 10 y 14 años (Núñez y González-Pienda, 1994; Núñez, González-Pienda, García, González y García, 1995) y universitarios (Valle et al., 2000), se encontró una taxonomía similar a la obtenida por Hayamizu y Weiner (1991).

Alonso (1991), a partir de sus investigaciones, en las que emplea otros instrumentos, agrupa las metas de los alumnos en cuatro categorías: metas relacionadas con la tarea, metas relacionadas con la autovaloración, metas relacionadas con la valoración social y metas relacionadas con la consecución de recompensas externas. Las metas relacionadas con la tarea se asocian con la motivación intrínseca; lo que se busca es incrementar la propia com- 
petencia, sentirse absorto por la naturaleza de la tarea y actuar con autonomía, es decir, no obligado a hacer algo. Las metas relacionadas con la autovaloración es una categoría estrechamente vinculada con el autoconcepto y la autoestima. Se busca alcanzar el éxito y experimentar las experiencias positivas de orgullo y satisfacción recibiendo una valoración positiva de la competencia actual, y evitar las experiencias negativas asociadas al fracaso. Las metas relacionadas con la valoración social, tienen que ver con la experiencia emocional que se deriva de las reacciones de personas significativas para el sujeto por su actuación; se busca la aprobación de su conducta académica y evitar el rechazo. Finalmente, las metas relacionadas con la consecución de recompensas externas, al igual que las anteriores, no se relacionan directamente con el aprendizaje, pero pueden utilizarse para favorecerlo. Están relacionadas con la consecución de premios y la evitación de castigos, es decir, incentivos externos (Alonso y Montero, 2001).

Lo que nos cuestionamos es si, en nuestra población estudiantil, al igual que en los estudios de Hayamizu, Ito y Yoshiazaki (1989) en Japón, de Hayamizu y Weiner (1991) en California y que en los trabajos realizados en nuestro país (Núñez y González-Pienda, 1994; Núñez, González-Pienda, García, González y García,1995; Valle et al., 2000), vamos a encontrar tres tipos de metas explicativas de la motivación o, incluso, podemos identificar más, como ocurre en los estudios de Alonso. Ya que, como apunta Marchesi (1999), el alumno no está motivado o desmotivado en abstracto, sino que lo está en función del significado que tiene para él el trabajo escolar. Los motivos a los que alude el alumno deberán entenderse a partir de sus experiencias previas, como resultado de la interacción que mantiene con los diferentes contextos en los que están presenten los aprendizajes académicos.

El objetivo general de nuestro trabajo es, pues, averiguar las metas que persiguen los alumnos en relación con los aprendizajes académicos, así como la relación de éstas con los diferentes patrones motivacionales. De un modo más particular nuestro trabajo se orienta a:

- Comprobar si las metas que persiguen los alumnos de nuestro ámbito en situación educativa responden o no a la tipología obtenida en otros contextos (metas de aprendizaje, de refuerzo social y de logro).

- Detectar si existen o no diferencias entre las metas de los alumnos de primaria y de secundaria.

- Valorar qué sentido tienen estas diferencias, en caso de producirse.

- Averiguar si los alumnos de secundaria perfilan más o menos sus metas que los de primaria.

- Establecer cómo se relacionan las metas de los estudiantes con las dimensiones causales de las atribuciones realizadas en situación educativa.

A tenor del objetivo general que hemos indicado anteriormente y de los objetivos específicos recién enunciados, en este estudio nos planteamos la comprobación de las siguientes hipótesis de trabajo:

Primera.- Existen metas de refuerzo social, de logro y de aprendizaje. Es decir, las metas de los alumnos de nuestra población estudiantil coinciden con las obtenidas en otras investigaciones llevadas a cabo en poblaciones distintas.

Segunda.- Del mismo modo que los alumnos más pequeños no discriminan entre esfuerzo y capacidad, podemos suponer que tampoco establecen diferencias entre los distintos tipos de metas. Es decir, hay diferencias entre las metas que señalan los 
alumnos de primaria (que serán más globales e indefinidas) y las que señalan los de secundaria (que serán más específicas y concretas).

Tercera.- Existe relación entre las metas y las dimensiones causales de las atribuciones más comunes para el rendimiento en contextos educativos (capacidad, esfuerzo, suerte, dificultad de la tarea, etc.). Es decir, los alumnos con metas de aprendizaje tenderán a la internalidad, a la estabilidad y a la controlabilidad y los alumnos con metas de refuerzo social y de logro propenderán a la externalidad, a la inestabilidad y a la incontrolabilidad al establecer las dimensiones de las atribuciones.

\section{Método}

\section{Muestra}

La población de la que se extrae la muestra la configuran los alumnos del tercer ciclo de Primaria (9-11 años) y de ambos ciclos de Secundaria Obligatoria (12-16 años) de la ciudad de Alicante que cursan sus estudios en centros públicos y concertados. La muestra se selecciona por muestreo aleatorio simple y la componen 722 sujetos con una edad media de 12,6 años $(\sigma=1,28)$. El $28 \%$ estudian primaria y el $72 \%$ secundaria. El $48,4 \%$ son chicos y el $51,6 \%$ restante son chicas.

\section{Variables e instrumentos}

a) Tipos de metas, que se evalúan con el cuestionario de metas de Hayamizu, Ito y Yohiazaki (1989), adaptado por Hayamizu y Weiner (1991), y traducido al castellano. Al sujeto se le ofrecen una serie de razones por las que se estudia y su tarea consiste en indicar, en una escala graduada del 1 al 5 , en la que 1 significa nunca y 5 significa siempre o todas las veces, los motivos por los que él estudia. En los estudios mencionados, el cuestionario muestra una fiabilidad (consistencia interna) entre 0,89 y 0,71 .

b) Dimensiones causales, que se evalúan con el cuestionario de dimensionalización causal de Hayamizu y Weiner (1991) traducido al castellano. El alumno ha de valorar las dimensiones de estabilidad (estable-inestable y temporal-permanente), de lugar (personal-medioambiental y externo-interno) y de controlabilidad (controlableincontrolable y no responsable-reponsable), en una escala bipolar de siete puntos, para una serie de adscripciones causales relativas al rendimiento académico (capacidad intelectual, falta de esfuerzo, dificultad del examen, mala explicación del profesor y mala suerte).

\section{Procedimiento}

Las variables son evaluadas, anónimamente, en los contextos del aula habitual de los alumnos y alumnas, indicando, expresamente, que las respuestas no tienen consecuencias sobre las calificaciones escolares y solicitando la máxima sinceridad. El cuestionario de metas y la escala de dimensionalización causal se responden en una única sesión. 


\section{Diseño}

El diseño, toda vez que no hay una manipulación intencional de las variables, se ajusta a las características de los diseños correlacionales básicos.

\section{Análisis}

Los datos se someten a análisis factoriales (por el método de extracción de componentes principales y de rotación Varimax), correlacionales ( $\mathrm{r}$ de Pearson) y de diferencias de medias para grupos independientes (prueba $t$ de Student). Se emplea, para ello, el paquete estadístico de programas SPSS (versión 9.0).

\section{Resultados}

Los resultados del análisis factorial, al que se someten las respuestas del cuestionario de metas, superan los criterios de significación estadística $(\mathrm{p}<0,001)$ en la prueba de esfericidad de Bartlett $\left(\chi^{2}=4691,235\right.$; g.l. $\left.=190 ; n=663\right)$ y la matriz de componentes rotados (la rotación converge en 5 iteraciones) arroja la existencia de tres factores cuyas saturaciones se muestran en la tabla número 1.

Como se aprecia en la misma, en el Factor I saturan los elementos comprendidos entre el 1 y el 8, que expresan razones tales como «es interesante resolver problemas», «disfruto descubriendo cuánto he mejorado», «deseo saber cosas» o «siento curiosidad». Este factor, vinculado a las metas de aprendizaje, da cuenta del 29,33\% de la varianza. El coeficiente de fiabilidad, consistencia interna alfa de Cronbach, para los elementos que integran el factor es $\mathrm{a}=0,8318$.

En el Factor II, los elementos que muestran una mayor carga factorial son los comprendidos entre el 15 y el 20, que se relacionan con metas del tipo «quiero sacar buenas notas», «quiero realizar estudios superiores», «deseo estar orgulloso de haber sacado buenas notas» o «quiero tener en el futuro un buen trabajo», es decir, metas relacionadas con el logro (aproximación al éxito y evitación del fracaso). Este factor explica el 12,94\% de la varianza total y el coeficiente de consistencia interna es muy similar al arrojado por los elementos del factor anterior $(\alpha=0,8245)$.

En el Factor III, los elementos que muestran una mayor saturación factorial son los comprendidos entre el 9 y el 14, que expresan motivos como «quiero ser elogiado», «quiero llamar la atención», «no quiero que se burlen de mí» o «no quiero que el profesor me tenga aversión», que son metas orientadas a la obtención de la aprobación o de refuerzo social. Este factor explica un $9,61 \%$ de la varianza total. El coeficiente de fiabilidad, consistencia interna alfa de Cronbach, para los elementos que integran el factor es $\alpha=0,8261$ (Tabla 1).

El total de la varianza explicada por los tres factores es $51,88 \%$ y la fiabilidad para el total de la escala (consistencia interna a de Cronbach) es igual a 0,8668.

Se hallan así tres tipos de metas que coinciden con las obtenidas por Hayamizu, Ito y Yohiazaki (1989) en Japón, por Hayamizu y Weiner (1991) en Estados Unidos y por Núñez y sus colaboradores $(1994,1995)$ en nuestro país, sin embargo, en nuestro caso, las metas 
TABLA 1: Saturaciones factoriales de los elementos del cuestionario de metas en cada uno de los factores obtenidos tras la rotación (método Varimax).

\begin{tabular}{|c|c|c|c|}
\hline \multirow{2}{*}{ Elementos del Cuestionario } & \multicolumn{3}{|c|}{ Matriz de componentes rotados } \\
\hline & Factor I & Factor II & Factor III \\
\hline $\begin{array}{l}\text { 1. } \ldots \text { es interesante resolver problemas. } \\
2 . \\
\text { 3. } \ldots \text { disfruto descubriendo cuánto he mejorado } \\
\text { 4. } \ldots \text { deseo saber cosas nuevas. } \\
5 . \\
\text { 5. } \ldots \text { me gusta el desafío de los problemas difíciles. } \\
6 . \\
\text { 7. } \ldots \text { siento curiosidad. } \\
\text { 8. } \ldots \text { me gusta emplear mi cabeza. }\end{array}$ & $\begin{array}{l}0,686 \\
0,711 \\
0,647 \\
0,711 \\
0,556 \\
0,648 \\
0,643 \\
0,596\end{array}$ & & \\
\hline 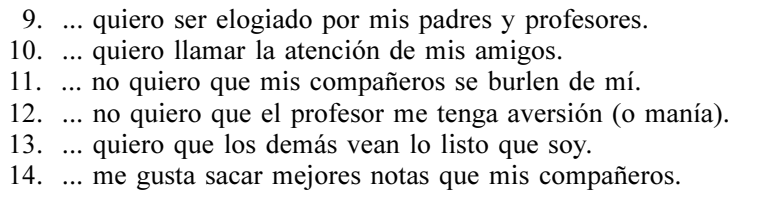 & & & $\begin{array}{l}0,551 \\
0,757 \\
0,736 \\
0,734 \\
0,782 \\
0,729\end{array}$ \\
\hline $\begin{array}{l}15 . \\
16 . \\
16 \text { quiero sacar buenas notas. } \\
\text { 17. ... no quiero suspender los exámenes finales. } \\
18 . \text {... quiero realizar estudios superiores. } \\
19 . \text {... quiero tener en el futuro un buen trabajo. } \\
20 .\end{array}$ & & $\begin{array}{l}0,771 \\
0,694 \\
0,612 \\
0,647 \\
0,761 \\
0,762\end{array}$ & \\
\hline
\end{tabular}

Factor I: Metas de aprendizaje; Factor II: Metas de logro; Factor III: Metas de refuerzo social.

de aprendizaje sí correlacionan, de modo estadísticamente significativo, con las metas de $\operatorname{logro}(r=0,456 ; p<0,001)$ y con las metas de refuerzo social $(r=0,339 ; p<0,001)$.

Los resultados de la prueba $t$ de Student de diferencias de medias para grupos independientes sin asumir la igualdad de las varianzas, en el que un grupo lo componen el alumnado de primaria $\left(n_{1}=176\right)$ y el otro lo integran los estudiantes de secundaria obligatoria $\left(\mathrm{n}_{2}=487\right)$, se muestran en la tabla número 2. Como se aprecia en la misma, existen diferencias estadísticamente significativas $(\mathrm{p}<0,005)$ en los tres tipos de metas (de aprendizaje, de logro y de refuerzo social) en función de la etapa educativa. El alumnado de primaria tiende a puntuar más alto en las tres metas que el de secundaria obligatoria (Tabla 2).

Finalmente, en la tabla número 3, se muestran las correlaciones (r de Pearson) existentes entre los tres tipos de metas (de aprendizaje, de logro y de refuerzo social) y las dimensiones causales de estabilidad, lugar y controlabilidad para las distintas atribuciones causales consideradas (capacidad, falta de esfuerzo, dificultad del examen, mala explicación del profesor y mala suerte).

En relación con la asociación existente entre los tres tipos de metas y las tres dimensiones causales de la atribución a la capacidad intelectual, los coeficientes de correlación nos indican que no son significativas ninguna de las correspondientes a las metas de aprendizaje, mientras que son estadísticamente significativas las relaciones entre el lugar y la controlabilidad con las metas de logro $(r=-0,117 ; \mathrm{p}=0,002$ y $\mathrm{r}=-0,091 ; \mathrm{p}=0,019$, respectivamente) y el lugar con las metas de refuerzo social $(r=0,136 ; p<0,001)$. 


\section{TABLA 2: Prueba t de Student para las diferencias de medias entre el alumnado de primaria y el de secundaria obligatoria en los tres tipos de metas (de aprendizaje, de logro y de refuerzo social).}

\begin{tabular}{|l|l|c|c|c|c|c|}
\hline \multicolumn{1}{|c|}{ Variables } & \multicolumn{1}{|c|}{ Grupo } & $\overline{\mathbf{x}}$ & $\mathbf{s}$ & $\mathbf{t}$ & g.l. & $\mathbf{p}$ \\
\hline \multirow{2}{*}{ Metas de aprendizaje } & Primaria & 29,972 & 6,244 & 8,29 & 314 & 0,000 \\
& Secundaria & 25,400 & 6,344 & & & \\
\hline \multirow{2}{*}{ Metas de logro } & Primaria & 27,693 & 3,277 & 5,74 & 433 & 0,000 \\
& Secundaria & 25,840 & 4,591 & & & \\
\hline \multirow{2}{*}{ Metas de refuerzo social } & Primaria & 17,119 & 7,104 & 2,96 & 266 & 0,003 \\
& Secundaria & 15,353 & 5,854 & & & \\
\hline
\end{tabular}

El grado de dependencia que muestran los tres tipos de metas con las tres dimensiones causales de la atribución a la falta de esfuerzo que merecen ser destacados, porque resultan estadísticamente significativos, según los coeficientes de correlación obtenidos, son los que se producen entre las metas de aprendizaje y las tres dimensiones causales $(r=0,090$; $\mathrm{p}=0,020 ; \mathrm{r}=-0,096 ; \mathrm{p}=0,013$ y $\mathrm{r}=-0,083 ; \mathrm{p}=0,033$, respectivamente) y entre las metas de logro y las tres dimensiones causales $(r=0,144 ; \mathrm{p}<0,001, \mathrm{r}=-0,138 ; \mathrm{p}<0,001$, $\mathrm{r}=-0,080 ; \mathrm{p}=0,04)$.

En cuanto a las dimensiones de la atribución a la dificultad del examen, la dimensión de estabilidad muestra correlaciones estadísticamente significativas con las metas de logro $(\mathrm{r}=0,077 ; \mathrm{p}=0,046)$ y con las metas de refuerzo social $(\mathrm{r}=-0,096 ; \mathrm{p}=0,013)$ y la dimensión de controlabilidad lo hace con las metas de aprendizaje $(r=-0.090 ; p=0,021)$ y con las metas de logro $(r=-0,079 ; p=0,043)$.

Por lo que concierne a las dimensiones causales de la adscripción a lo mal que explica el profesor, ninguna de ellas correlaciona, de un modo estadísticamente significativo, con las metas consideradas.

Y, en último lugar, la dimensión de estabilidad de las atribuciones a la mala suerte correlaciona, de manera estadísticamente significativa, con las metas de aprendizaje $(\mathrm{r}=0,140 ; \mathrm{p}<0,001)$ y con las metas de logro $(\mathrm{r}=0,180 ; \mathrm{p}<0,001)$, asimismo lo hace la dimensión de controlabilidad con las metas de logro $(\mathrm{r}=0,109 ; \mathrm{p}=0,005)$ y con las de refuerzo social ( $\mathrm{r}=-0,137 ; \mathrm{p}<0,001)$ (Tabla 3).

\section{Discusión}

Los resultados del análisis factorial descubren la existencia de tres factores: el primer factor está vinculado a las metas de aprendizaje, el segundo implica metas relacionadas con el logro y el tercero metas orientadas a la obtención de la aprobación o del refuerzo social.

Hemos encontrado tres tipos de metas, al igual que ocurre en trabajos anteriores como los de Hayamizu, Ito y Yoshiazaki (1989), de Hayamizu y Weiner (1991) y que en trabajos realizados en nuestro país (Núñez y González-Pienda, 1994; Núñez, González-Pienda, García, González y García, 1995). 


\section{TABLA 3: Correlaciones entre los tres tipos de metas \\ (de aprendizaje, de logro y de refuerzo social) y las dimensiones causales percibidas en cada atribución.}

\begin{tabular}{|c|c|c|c|}
\hline $\begin{array}{c}\text { Dimensiones de las } \\
\text { adscripciones causales }\end{array}$ & Aprendizaje & Logro & $\begin{array}{c}\text { Refuerzo } \\
\text { social }\end{array}$ \\
\hline $\begin{array}{l}\text { Capacidad intelectual } \\
\text { - Estabilidad } \\
\text { - Lugar de control } \\
\text { - Controlabilidad }\end{array}$ & $\begin{array}{r}0,057 \\
-0,059 \\
-0,047\end{array}$ & $\begin{array}{l}0,061 \\
-0,117^{* *} \\
-0,091 *\end{array}$ & $\begin{array}{l}0,023 \\
0,136^{* *} \\
-0,069\end{array}$ \\
\hline $\begin{array}{l}\text { Falta de esfuerzo } \\
\text { - Estabilidad } \\
\text { - Lugar de control } \\
\text { - Controlabilidad }\end{array}$ & $\begin{array}{r}0,090^{*} \\
-0,096^{*} \\
-0,083^{*}\end{array}$ & $\begin{array}{r}0,144 * * \\
-0,138^{* *} \\
-0,080^{*}\end{array}$ & $\begin{array}{r}-0,059 \\
0,057 \\
0,064\end{array}$ \\
\hline $\begin{array}{l}\text { Dificultad del examen } \\
\text { - Estabilidad } \\
\text { - Lugar de control } \\
\text { - Controlabilidad }\end{array}$ & $\begin{array}{l}0,062 \\
-0,054 \\
-0,090^{*}\end{array}$ & $\begin{array}{l}0,077^{*} \\
-0,070 \\
-0,079 * \\
\end{array}$ & $\begin{array}{r}-0,096^{*} \\
0,019 \\
-0,072 \\
\end{array}$ \\
\hline $\begin{array}{l}\text { Mal que explica el profesor } \\
\text { - Estabilidad } \\
\text { - Lugar de control } \\
\text { - Controlabilidad }\end{array}$ & $\begin{array}{r}0,075 \\
0,030 \\
-0,024\end{array}$ & $\begin{array}{l}0,014 \\
0,008 \\
0,033\end{array}$ & $\begin{array}{r}0,010 \\
0,036 \\
-0,025\end{array}$ \\
\hline $\begin{array}{l}\text { Mala suerte } \\
\text { - Estabilidad } \\
\text { - Lugar de control } \\
\text { - Controlabilidad }\end{array}$ & $\begin{array}{c}0,140^{* *} \\
-0,011 \\
0,016\end{array}$ & $\begin{array}{l}0,180 * * \\
0,030 \\
0,109 *\end{array}$ & $\begin{array}{l}-0,012 \\
-0,016 \\
-0,137^{* *}\end{array}$ \\
\hline
\end{tabular}

$*: \mathrm{p}<0,05 ; \quad * *: \mathrm{p}<0,001$.

Los resultados obtenidos permiten confirmar nuestra primera hipótesis, en la que queríamos comprobar la coincidencia de las metas de los alumnos de nuestra población estudiantil con los tres tipos de metas para los aprendizajes académicos obtenidas en las investigaciones anteriormente mencionadas. Hemos encontrado que las metas que tienen los alumnos para los aprendizajes académicos pueden agruparse en los mismos tres tipos de metas obtenidas en contextos académicos y culturales distintos al nuestro (de aprendizaje, de logro y de refuerzo social).

Ahora bien, en nuestra población sí se da una relación entre las metas de aprendizaje y las metas de logro y de refuerzo social. Es decir, los alumnos no discriminan tres tipos de metas independientes, sino que consideran que entre ellas hay elementos comunes que las relacionan entre sí.

En estudios anteriores no se encontraba una relación entre las metas de aprendizaje y las de logro ni tampoco entre las de aprendizaje y las de refuerzo social, aunque sí existía relación entre las metas de logro y las de refuerzo social. Es decir, los alumnos discriminaban claramente los tipos de metas, no encontrando la existencia significativa de elementos comunes entre las metas de aprendizaje, que al parecer, no tenían nada que ver con las metas 
de ejecución (de logro y de refuerzo social). Sin embargo no tenían tan clara la independencia entre los dos tipos de metas de ejecución ya que se relacionaban significativamente. En nuestro caso, sin embargo, se aprecia cierto grado de asociación o de dependencia entre los tres tipos de metas, es decir, no son independientes entre sí.

En la segunda hipótesis aventuramos la existencia de diferencias entre las metas que señalan los estudiantes de primaria y los de secundaria. Las diferencias se justificaban al suponer que los alumnos más pequeños no discriminarían entre los distintos tipos de metas. Los resultados indican que, efectivamente, existen diferencias significativas entre los alumnos de primaria y los de secundaria en los tres tipos de metas. Las puntuaciones medias son más altas en los tres casos en los estudiantes de primaria que en los de secundaria. Parece ser que los alumnos de primaria justifican su conducta ante los aprendizajes académicos centrándose con mayor intensidad en estas metas, mientras que los alumnos de secundaria muestran unas puntuaciones más bajas. Los alumnos de primaria explican la motivación intrínseca por las tareas escolares y la motivación extrínseca en los refuerzos externos académicos y sociales, quizás asociando éxito a premios y recompensa social-escolar, y fracaso a castigo y desaprobación social. Por otra parte, los alumnos de secundaria, aunque delimitan las mismas metas, puntúan en ellas más moderadamente que los de primaria y no establecen de forma tan ajustada esas asociaciones; quizás encuentran otras razones a partir de las cuales pueden explicar mejor, o incluso justificar, su conducta hacia las tareas escolares. No se olvide que la «O» de las siglas E.S.O. significa «obligatoria» y que ningún elemento de la escala empleada afirma: «estudio porque me obligan». En todo caso, a pesar de las diferencias significativas obtenidas, nuestra hipótesis se ve desconfirmada ya que los alumnos de primaria sí discriminan y diferencian los tres tipos de metas y, además, alcanzan en ellas puntuaciones más elevadas que los de secundaria.

En relación a la tercera de nuestras hipótesis, los resultados indican que no se da una correlación significativa entre las metas de aprendizaje y las tres dimensiones causales de la atribución a la capacidad intelectual, lo que contradice el modelo de Dweck (1986). Recordemos que éste plantea una relación entre metas de aprendizaje y las metas de rendimiento con la capacidad intelectual en el sentido siguiente: los sujetos con metas de aprendizaje consideran que la capacidad intelectual es un rasgo estable y controlable, mientras que los alumnos con metas de rendimiento consideran que es un rasgo fijo o estable. La ausencia de correlación significativa obtenida en este trabajo cuestiona tales afirmaciones.

Sí se da una correlación entre las metas de logro y las dimensiones de lugar y controlabilidad, siendo esta correlación negativa, es decir, a medida que aumentan las explicaciones de logro, disminuye la externalidad y la controlabilidad asignada a la capacidad intelectual. Los sujetos que puntúan alto en metas de logro consideran que el éxito o el fracaso en sus tareas escolares depende de factores internos y controlables. Según los trabajos de Dweck, los sujetos con metas de logro consideran que la capacidad intelectual es estable e incontrolable, de manera que el sujeto no puede hacer nada para modificarla. Cuando el sujeto afronta el aprendizaje con metas de logro, considera que la capacidad intelectual es un rasgo interno e incontrolable, es decir, el sujeto no puede hacer nada para modificar esta capacidad o para aumentarla, por lo que su motivación para estudiar disminuye. En nuestro caso, los alumnos establecen la relación con el lugar (interno) y la controlabilidad (controlable) es, por tanto, un rasgo interno que pueden controlar. Se atribuyen, de esta forma, la capacidad para modificarla, para ellos no se trata de un rasgo fijo sobre el que no tienen ningún control. En este punto los resultados no son coincidentes con los aportados por 
Dweck. También se ha obtenido una correlación positiva en los sujetos con metas de refuerzo social para la adscripción a la capacidad intelectual con la dimensión de lugar. Los alumnos con metas de refuerzo social consideran que la capacidad intelectual es un factor externo y que no depende de ellos.

En cuanto a la atribución a la falta de esfuerzo se ha hallado dependencia entre los tres tipos de metas y las tres dimensiones causales. Se ha encontrado una correlación positiva entre las metas de aprendizaje y las dimensiones de lugar y estabilidad y, en sentido negativo, con la controlabilidad.

Los alumnos con metas de aprendizaje consideran que la falta de esfuerzo es inestable y es un rasgo interno al sujeto. Si el alumno considera que la falta de esfuerzo es inestable, es decir, varía de una situación a otra, y es interna, es decir, depende de él, es él quien va a ejercer la decisión de cambiar esa falta de esfuerzo según su propia voluntad. En este sentido, la intervención del educador podrá ir dirigida a cambiar esa falta de esfuerzo y a incentivar al alumno para que realice dicho cambio puesto que, según el alumno, el cambio depende de él mismo y no de los demás.

Los alumnos con metas de logro consideran que la falta de esfuerzo es inestable e interna, al igual que se asume en las aportaciones de Weiner (1986). En este sentido, al igual que para los alumnos con metas de aprendizaje, el alumno con metas de logro es consciente que esa falta de esfuerzo es interna, es decir, está en él mismo, y es inestable por lo que la puede modificar en cada ocasión. Sin embargo, los resultados indican que las metas de refuerzo social no se relacionan con las dimensiones causales de la falta de esfuerzo.

En cuanto a las dimensiones causales de la atribución a la dificultad del examen, se han encontrado relaciones con las metas de aprendizaje y con las de refuerzo social. Los alumnos con metas de aprendizaje consideran que la dificultad del examen es controlable, pueden controlar que la dificultad del examen aumente o disminuya; los alumnos con metas de refuerzo social creen que la dificultad del examen es estable (siempre es la misma). Sin embargo, para los alumnos con metas de logro no existe ninguna relación significativa entre la dificultad del examen y las dimensiones causales. Para los alumnos con metas de aprendizaje, la dificultad del examen se puede controlar, por ejemplo, trabajando más para aprender con mayor profundidad los contenidos y hacer que disminuya la dificultad al adquirir de forma adecuada los conocimientos que la realización del examen exige.

Los resultados indican que, efectivamente, tanto para los alumnos con metas de aprendizaje, de logro y de refuerzo social, existe relación entre la atribución a la suerte y las dimensiones causales. En el sentido que los alumnos con metas de aprendizaje opinan que la mala suerte es inestable y que varía de una situación a otra (no será siempre la misma, ni se repetirá para cada situación), la suerte no está asignada a una persona siempre que realiza una actividad determinada. Es decir, el éxito o el fracaso en las tareas de aprendizaje no va a estar siempre marcado por la mala suerte o por la buena suerte: dependerá de cada ocasión.

Los alumnos con metas de logro consideran que la suerte es un factor inestable e incontrolable, lo que es coherente con el modelo de Weiner (1986). Para ellos, la suerte depende de cada situación particular en la que se encuentran aunque no la pueden controlar y no la pueden modificar. Esto no influirá negativamente en su motivación ya que la suerte variará de una situación a otra (una veces será buena, otras veces será mala). 
Los alumnos con metas de refuerzo social piensan que la suerte es controlable. Si es un factor que se puede controlar, el alumno cree que puede ejercer su influencia sobre la suerte $\mathrm{y}$, de este modo, puede estar motivado para estudiar ya que mediante su acción puede modificar positivamente la suerte.

En cuanto a la atribución a lo mal que explica el profesor no hemos hallado ningún tipo de relación entre los tres tipos de metas y las tres dimensiones causales.

En resumen, de todo lo anterior se pueden extraer tres conclusiones de interés. Éstas son las siguientes:

a) Los alumnos y alumnas de nuestro contexto afrontan las situaciones de aprendizaje con tres tipos de metas (de aprendizaje, de logro y de refuerzo social) que son coincidentes con las obtenidas en otros contextos distintos. Se replican, así, los hallazgos de Hayamizu, Ito y Yohiazaki (1989), de Hayamizu y Weiner (1991) y de Núñez et al. (1994, 1995). Si bien, las metas de parendizaje parecen depender de las de ejecución (logro y refuerzo social) al contrario de lo obtenido en los estudios mencionados.

b) Las metas del alumnado de primaria coinciden con las del alumnado de secundaria, aunque se observan diferencias entre ellos: los de primaria puntúan más alto que los de secundaria en los tres tipos de metas.

c) Las metas que el alumnado persigue en situación de aprendizaje se relacionan con las dimensiones causales de lugar, estabilidad y controlabilidad. No obstante, el sentido y la magnitud de estas relaciones se ven mediadas por el tipo de causa que se dimensionaliza, no existiendo un patrón generalizable a todos ellas.

\section{Referencias bibliográficas}

Alonso, J. y Montero, I. (2001). Orientación motivacional y estrategias motivadoras en el aprendizaje escolar. En C. Coll, J. Palacios y A. Marchesi (Comp.), Desarrollo psicológico y educación, 2. Psicología de la educación escolar (pp. 259-283). Madrid: Alianza.

Alonso, J. (1991). Motivación y aprendizaje en el aula. Cómo enseñar a pensar. Madrid: Santillana.

Ames, C.; Ames, R. y Felker, D. W. (1977). Effects of competitive reward structure and valence of outcome on children's achievement attributions. Journal of Educational Psychology, 69, 1-8.

Ames, C. (1984). Competitive, cooperative, and individualistic goal structures: A motivational analysis. En R. Ames y C. Ames (Eds.), Research on motivation in education: Vol. 1 Student motivation. Nueva York: Academic Press.

Bzuneck, J. A. (1999). Uma abordagem socio-cognitivista à motivaçao do aluno: a teoria de metas de realizaçao. Psico-USF, 4 (2), 51-66.

Castejón J. L.; Navas, L. y Sampascual, G. (1993). Modelos estructurales sobre la teoría atribucional de la motivación. Revista de Psicología General y Aplicada, 46 (3), 293-305.

Cervelló, E. M. y Santos-Rosa, F. J. (2000). Motivación en las clases de educación física: un estudio de la perspectiva de las metas de logro en el contexto educativo. Revista de Psicología del Deporte, 9 (1-2), 51-70.

Cevelló, E. M.; Escartí, A. y Balagué, G. (1999). Relaciones entre la orientación de meta disposicional y la satisfacción con los resultados deportivos, las creencias sobre las causas de éxito en deporte y la diversión con la práctica deportiva. Revista de Psicología del Deporte, 8 (1), 7-19. 
Dweck, C. S. (1986). Motivational processes affecting learning. American Psychologist, 41, $1040-$ 1048 .

Dweck, C. S. y Legget, E. (1989). A social cognitive approach to motivation and personality. Psychological Review, 95, 256-273.

Fontaine, G. (1974). Social comparison and some determinants of expected personal control and expected performance in a novel task situation. Journal of Personality and Social Psychology, 29, 487-496.

González-Pienda, J. A.; Núñez, J. C.; González-Pumariega, S.; Álvarez, L.; Roces, C.; García, M.; González, P.; Cabanach, R. G. y Valle, A. (2000). Autoconcepto, proceso de atribución causal y metas académicas en niños con y sin dificultades de aprendizaje. Psicothema, 12 (4), 548-556.

Hayashi, T. y Yamachi, H. (1974). Causal attributional judgments for achievement-related events. Japanese Psychological Research, 16, 40-49.

Hayamizu,T.; Ito, A., y Yohiazaki, K. (1989). Cognitive motivational process mediated by achievement goal tendencies. Japonese Research, 31, 179-189.

Hayamizu, T. y Weiner, B. (1991). A test of Dweck's model of achievement goals are related to perceptions of ability. Journal of Experimental Education, 59, 226-234.

Heilman, M. E. y Guzzo, R. A. (1978). The perceived causes of work sucess as a mediator of sex discriminations in organizations. Organizational Behavior and Human Performance, 21, 346-357.

Heyman G. D. y Dweck, C. S. (1992). Achievement goal and intrisic motivation: Their relation and their role in adaptative motivation. Motivation and Emotion, 16, 231-247.

Inagi, T. (1977). Causal adscription and expectancy of sucess. Japanesc Psychological Research, 19, 23-30.

Kojima, M. (1984). A study of causal atribution in achievement-related behabior: An analysis both in the experiemental and in the educational setting. Journal of Child Development, 20, 20-30.

Marchesi, A. (1999). Los alumnos con escasa motivación para aprender. En A. Marchesi, C. Coll y J. Palacios (Coord.), Desarrollo psicológico y educación. 3. Trastornos del desarrollo y necesidades educativas especiales (pp. 183-208). Madrid: Alianza Editorial.

Navas, L.; Castejón, J. L. y Sampascual, G. (1996). Un modelo causal del rendimiento académcio en la ESO desde perspectivas cognitivo motivacionales. Cartagena: Ediciones Faro.

Navas, L.; Castejón, J. L. y Sampascual, G. (2000). Un contraste del modelo atribucional de la motivación de Weiner en contextos educativos. Revista de Psicología Social, 15 (2), 69-85.

Núñez, J. C. y González-Pienda J. A. (1994). Determinantes del rendimiento académico. Oviedo: Servicio de Publicaciones de la Universidad de Oviedo.

Núñez, J. C.; González-Pienda, J. A.; González-Pumariaga, S.; García M. S. y García, S. I. (1995). Estrategias de aprendizaje en alumnos de 10 a 14 años y su relación con los procesos de atribución causal, el autoconcepto y las metas de estudio de los alumnos. Revista Galega de Psicopedagogía, 10-11, 1134-1114.

Montero, I. y Alonso, J. (1992). Achievement motivation in high school: contrasting theoretical models in the classroom. Learning and Instruction, 2, 43-57.

Sampascual, G.; Navas, L. y Castejón, J. L. (1994). Procesos atribucionales en la educación secundaria obligatoria: Un análisis para la reflexión. Revista de Psicología General y Aplicada, 47, 4, 449-459.

Valle, A. y Gómez, M. L. (1997). Motivación y estrategias de aprendizaje significativo. Boletín de Psicología, 56, 37-51.

Valle, A.; González, R.; Núñez, J. C.; Súarez, J. M.; Piñeiro, I. y Rodríguez, S. (2000). Enfoques de aprendizaje en estudiantes universitarios. Psicothema, 12 (3), 368-375. 
Weiner, B. (1986). An attributional theory of motivation and emotion. Nueva York: Springer-Verlag. Weiner, B. (1992). Human motivation. Metaphors, theories and research. California: Sage.

Weiner, B. (1995). Judgments of responsability. A foundation for a theory of social conduct. Nueva York: Guilford Press.

Fecha de recepción: 5-II-01

Fecha de revisión: 10-IX-01

Fecha de aceptación: 5-II-02 


\section{ANEXO I \\ Cuestionario de Metas (Hayamizu, Ito y Yohiazaki, 1989; \\ Hayamizu y Weiner, 1991).}

\section{Este cuestionario es anónimo y no tiene consecuencias en sus calificaciones. Responda lo que piensa de verdad. SEA SINCERO}

Por favor complete los datos siguientes:

\begin{tabular}{|ll|}
\hline Edad ___ Etapa educativa que estudia & Curso \\
Nombre del centro & Localidad \\
Marque con una cruz lo que corresponda: Chico $\square$ Chica $\square$
\end{tabular}

A continuación aparecen diversas razones por las que la gente estudia. Junto a cada razón aparecen unos números del 1 al 5. Se trata de indicar, rodeando con un círculo el número correspondiente, el grado en el que cada motivo influye en por qué estudias tú. Para ello hay que tener en cuenta que:

- 1 significa nunca.

- 2 significa rara vez (muy pocas veces).

- 3 significa algunas veces.

- 4 significa con frecuencia (casi siempre).

- 5 significa siempre (todas las veces).

Nunca Siempre

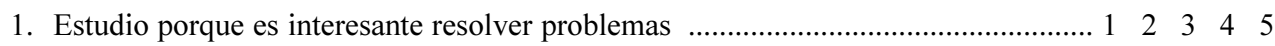

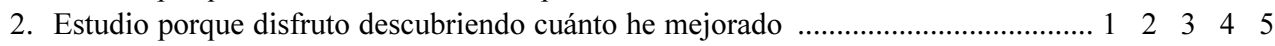

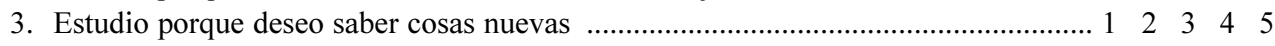

4. Estudio porque me gusta el desafío de los problemas difíciles .............................. $1 \begin{array}{llllll}1 & 2 & 3 & 4 & 5\end{array}$

5. Estudio porque me siento bien cuando supero obstáculos y fracasos ………............ $1 \begin{array}{lllll}1 & 2 & 3 & 4 & 5\end{array}$

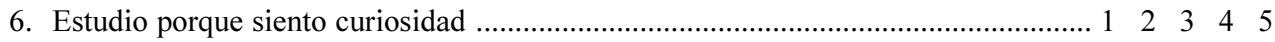

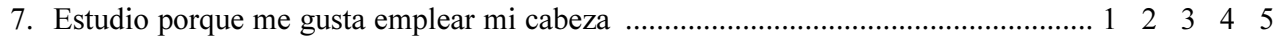

8. Estudio porque me da alegría cuando puedo resolver un problema difícil ................ $1 \begin{array}{llllll}1 & 2 & 3 & 4 & 5\end{array}$

9. Estudio porque quiero ser elogiado (felicitado) por mis padres y profesores ............ $1 \begin{array}{llllll}1 & 2 & 3 & 4 & 5\end{array}$

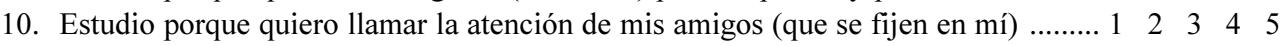

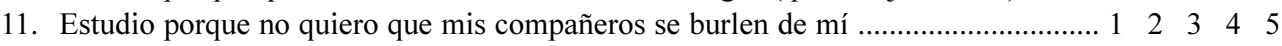

12. Estudio porque no quiero que el profesor me tenga aversión (o manía)

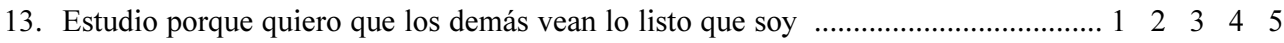

14. Estudio porque me gusta sacar mejores notas que mis compañeros ………….......... $1 \begin{array}{llllll}1 & 2 & 3 & 4 & 5\end{array}$

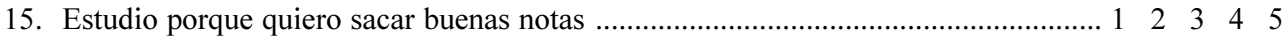

16. Estudio porque deseo estar orgulloso de haber sacado buenas notas ......................... $1 \begin{array}{lllll}1 & 2 & 3 & 4 & 5\end{array}$

17. Estudio porque no quiero suspender los exámenes finales ....................................... $1 \begin{array}{llllll}1 & 2 & 3 & 4 & 5\end{array}$

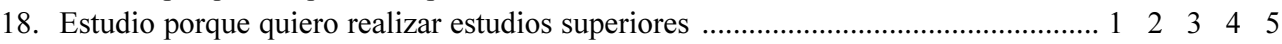

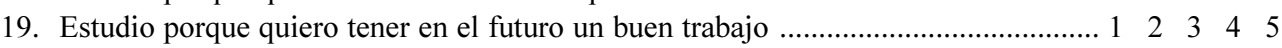

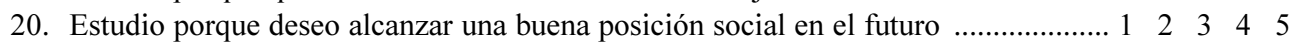




\section{ANEXO II \\ Cuestionario de dimensiones causales (Hayamizu y Weiner, 1991).}

En su rendimiento académico y en las calificaciones que obtenga pueden influir diversos factores o causas. Normalmente se obtienen malas notas o se suspende por tener poca capacidad intelectual (ser poco inteligente), por no esforzarse lo suficiente, porque el examen ha sido difícil, porque el profesor no explica bien o porque se ha tenido mala suerte.

¿Qué propiedades cree que tienen esas causas? Para responder hay que valorar seis características de cada una de esas posibles causas en una escala de siete puntos según se acerquen más a un extremo de la escala o al otro. Rodee con un círculo el número que corresponda.

\section{La capacidad intelectual es:}

Estable (no cambia con el tiempo ni en las situaciones)

Personal (está dentro de la persona)

Controlable (que uno lo hace a su voluntad)

Temporal (no sigue igual, es pasajero)

Externa (fuera de usted)

No responsable (no depende de uno)

\section{La falta de esfuerzo es:}

Estable (no cambia con el tiempo ni en las situaciones)

Personal (está dentro de la persona)

Controlable (que uno lo hace a su voluntad)

Temporal (no sigue igual, es pasajero)

Externa (fuera de usted)

No responsable (no depende de uno)

\section{La dificultad del examen es:}

Estable (no cambia con el tiempo ni en las situaciones) $\begin{array}{llllllll}1 & 2 & 3 & 4 & 5 & 6 & 7 & \text { Inestable (cambia con el tiempo y en }\end{array}$ las situaciones)

$\begin{array}{llllllll}1 & 2 & 3 & 4 & 5 & 6 & 7 & \text { Medioambiental (está fuera de la }\end{array}$ persona)

$\begin{array}{llllllll}1 & 2 & 3 & 4 & 5 & 6 & 7 & \text { Incontrolable (que uno no puede }\end{array}$ hacerlo a su voluntad)

$\begin{array}{llllllll}1 & 2 & 3 & 4 & 5 & 6 & 7 & \text { Permanente (sigue igual siempre, no }\end{array}$ se pasa)

$\begin{array}{llllllll}1 & 2 & 3 & 4 & 5 & 6 & 7 & \text { Interna (dentro de usted) }\end{array}$

$\begin{array}{llllllll}1 & 2 & 3 & 4 & 5 & 6 & 7 & \text { Responsable (depende de uno) }\end{array}$

$\begin{array}{llllllll}1 & 2 & 3 & 4 & 5 & 6 & 7 & \text { Inestable (cambia con el tiempo y en }\end{array}$ las situaciones)

$\begin{array}{llllllll}1 & 2 & 3 & 4 & 5 & 6 & 7 & \text { Medioambiental (está fuera de la }\end{array}$ persona)

$\begin{array}{llllllll}1 & 2 & 3 & 4 & 5 & 6 & 7 & \text { Incontrolable (que uno no puede }\end{array}$ hacerlo a su voluntad)

$\begin{array}{llllllll}1 & 2 & 3 & 4 & 5 & 6 & 7 & \text { Permanente (sigue igual siempre, no }\end{array}$ se pasa)

$\begin{array}{llllllll}1 & 2 & 3 & 4 & 5 & 6 & 7 & \text { Interna (dentro de usted) }\end{array}$

$\begin{array}{llllllll}1 & 2 & 3 & 4 & 5 & 6 & 7 & \text { Responsable (depende de uno) }\end{array}$

$\begin{array}{llllllll}1 & 2 & 3 & 4 & 5 & 6 & 7 & \text { Inestable (cambia con el tiempo y en }\end{array}$ las situaciones) 
Personal (está dentro de la persona) $\quad \begin{array}{llllllll}1 & 2 & 3 & 4 & 5 & 6 & 7 & \text { Medioambiental (está fuera de la }\end{array}$ persona)

$\begin{array}{llllllllll}\text { Controlable (que uno lo hace a su } & 1 & 2 & 3 & 4 & 5 & 6 & 7 & \text { Incontrolable (que uno no puede }\end{array}$ voluntad) hacerlo a su voluntad)

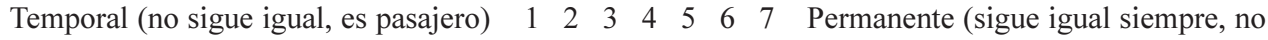
se pasa)

Externa (fuera de usted)

$\begin{array}{llllllll}1 & 2 & 3 & 4 & 5 & 6 & 7 & \text { Interna (dentro de usted) }\end{array}$

No responsable (no depende de uno)

$\begin{array}{llllllll}1 & 2 & 3 & 4 & 5 & 6 & 7 & \text { Responsable (depende de uno) }\end{array}$

\section{Lo mal que explica el profesor es:}

Estable (no cambia con el tiempo ni en las situaciones)

$\begin{array}{llllllll}1 & 2 & 3 & 4 & 5 & 6 & 7 & \text { Inestable (cambia con el tiempo y en }\end{array}$ las situaciones)

Personal (está dentro de la persona)

$\begin{array}{llllllll}1 & 2 & 3 & 4 & 5 & 6 & 7 & \text { Medioambiental (está fuera de la }\end{array}$ persona)

Controlable (que uno lo hace a su voluntad)

$\begin{array}{llllllll}1 & 2 & 3 & 4 & 5 & 6 & 7 & \text { Incontrolable (que uno no puede }\end{array}$ hacerlo a su voluntad)

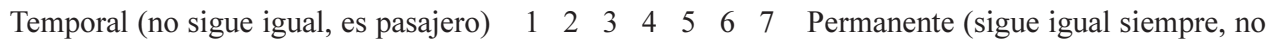
se pasa)

Externa (fuera de usted)

$\begin{array}{llllllll}1 & 2 & 3 & 4 & 5 & 6 & 7 & \text { Interna (dentro de usted) }\end{array}$

No responsable (no depende de uno)

$\begin{array}{llllllll}1 & 2 & 3 & 4 & 5 & 6 & 7 & \text { Responsable (depende de uno) }\end{array}$

\section{La mala suerte es:}

Estable (no cambia con el tiempo ni en las situaciones)

$\begin{array}{llllllll}1 & 2 & 3 & 4 & 5 & 6 & 7 & \text { Inestable (cambia con el tiempo y en }\end{array}$ las situaciones)

Personal (está dentro de la persona)

$\begin{array}{llllllll}1 & 2 & 3 & 4 & 5 & 6 & 7 & \text { Medioambiental (está fuera de la }\end{array}$ persona)

Controlable (que uno lo hace a su voluntad)

$\begin{array}{llllllll}1 & 2 & 3 & 4 & 5 & 6 & 7 & \text { Incontrolable (que uno no puede }\end{array}$ hacerlo a su voluntad)

Temporal (no sigue igual, es pasajero)

Externa (fuera de uno)

No responsable (no depende de uno)

$\begin{array}{llllllll}1 & 2 & 3 & 4 & 5 & 6 & 7 & \text { Interna (dentro de uno) }\end{array}$

$\begin{array}{llllllll}1 & 2 & 3 & 4 & 5 & 6 & 7 & \text { Responsable (depende de uno) }\end{array}$ 